\title{
Twist-2 transverse momentum dependent distributions
}

\section{Daniel Gutiérrez-Reyes*}

Departamento de Física Teórica, Universidad Complutense de Madrid(UCM), 28040 Madrid, Spain

E-mail: dangut01@ucm.es

\section{Ignazio Scimemi}

Departamento de Física Teórica, Universidad Complutense de Madrid(UCM), 28040 Madrid, Spain

E-mail: ignazios@ucm.es

\section{Alexey A. Vladimirov}

Institut für Theorische Physik, Universität Regensburg, D-93040 Regensburg, Germany

E-mail: vladimirov.aleksey@gmail.com

\begin{abstract}
The factorization theorem for Drell-Yan and semi-inclusive DIS holds for all leading twist transverse momentum distributions. However a QCD perturbative calculation shows several important characteristics of spin-dependent distributions. We consider all the different spin-dependent distributions which can be matched onto integrated twist- 2 functions, focusing on the Helicity, Transversity and Pretzelosity distributions. The pretzelosity case is specially relevant because, using a direct perturbative calculation at one loop, we obtain a null result which agrees with the experimental measurements.
\end{abstract}

13th International Symposium on Radiative Corrections 24-29 September, 2017

St. Gilgen, Austria

\footnotetext{
*Speaker.
} 


\section{Introduction}

The transverse momentum dependent (TMD) factorization theorems formulated in [1, 2, 3, 4] provide a self-contained definition of TMD operators which can be considered individually without referring to a scattering process. This is because the factorization theorems allow a consistent treatment of rapidity divergences in the definition of spin (in)dependent TMD distributions. We focus on the evaluation of the large- $q_{T}$ (or small- $b$ ) matching of the TMD operators on the corresponding integrated functions. The matching coefficients resulting are practically very important because they serve as an initial imput to many models and phenomenological ansatzes for TMD distributions. The unpolarized TMD distribution is the most studied case at the next-to-leadingorder (NLO) [1, 5, 2, 4, 6, 7, 8] and the next-to-next to leading order (NNLO) [9, 10, 11, 12]. This paper is dedicated to show results for polarized distributions obtained in a systematic way as in $[13,14]$ for helicity, transversity and pretzelosity distributions.

The quark and gluon components of the generic TMD operators are

$$
\begin{aligned}
\Phi_{i j}(x, \boldsymbol{b}) & =\int \frac{d \lambda}{2 \pi} e^{-i x p^{+} \lambda} \bar{q}_{i}(\lambda n+\boldsymbol{b}) \mathscr{W}(\lambda, \boldsymbol{b}) q_{j}(0), \\
\Phi_{\mu v}(x, \boldsymbol{b}) & =\frac{1}{x p^{+}} \int \frac{d \lambda}{2 \pi} e^{-i x p^{+} \lambda} F_{+\mu}(\lambda n+\boldsymbol{b}) \mathscr{W}(\lambda, \boldsymbol{b}) F_{+v}(0),
\end{aligned}
$$

where $n$ is the lightlike vector used in lightcone coordinates and $\mathscr{W}(\lambda, \boldsymbol{b})$ is the gauge link made of Wilson lines. Its staples contour results in the rapidity divergences. They are removed by the proper rapidity renormalization factor $R$, which is built from the TMD soft factor,

$$
S(\boldsymbol{b})=\frac{\operatorname{Tr}_{\text {color }}}{N_{c}}\left\langle 0\left|\left[S_{n}^{T \dagger} \tilde{S}_{\bar{n}}^{T}\right](\boldsymbol{b})\left[\tilde{S}_{\bar{n}}^{T \dagger} S_{n}^{T}\right](0)\right| 0\right\rangle,
$$

where $S_{n}$ and $\tilde{S}_{\bar{n}}$ stand for soft Wilson lines (for the precise definition of Wilson lines see e.g. [10]). The structure of the factor $R$ depend on the rapidity regularization scheme. In our case the scheme used is the $\delta$-regularization scheme, which is based in a regularization of the rapidity divergences directly from the operators, by using decreasing exponentials with the rapidity regulator, $\delta$, as

$$
\begin{aligned}
& W_{n}=P \exp \left(-i g \int_{0}^{\infty} d \sigma(n \cdot A)(n \sigma)\right) \rightarrow W_{n}=P \exp \left(-i g \int_{0}^{\infty} d \sigma(n \cdot A)(n \sigma) e^{-\delta x \sigma}\right), \\
& S_{n}=P \exp \left(-i g \int_{0}^{\infty} d \sigma\left(n \cdot A_{s}\right)(n \sigma)\right) \rightarrow S_{n}=P \exp \left(-i g \int_{0}^{\infty} d \sigma\left(n \cdot A_{s}\right)(n \sigma) e^{-\delta \sigma}\right) .
\end{aligned}
$$

This scheme violates gauge properties of Wilson lines by power supressed in $\delta$ terms, i.e. only the calculation at $\delta \rightarrow 0$ is legitimate. Also this regularization makes the non-abelian exponentiation of the soft function satisfied at all orders. For another part, in this scheme the zero-bin contribution is equal to the soft factor, obtaining a simple expression for $R$,

$$
R=\frac{\sqrt{S(\boldsymbol{b})}}{\text { zero-bin }} \stackrel{\delta-\text { reg. }}{\longrightarrow} R_{\delta \text {-reg. }}=\frac{1}{\sqrt{S(\boldsymbol{b})}} .
$$


The rapidity divergence regularization scheme affects to the quantities related to the regularization and renormalization of the rapidity divergences, but does not affect to the rapidity-divergencesfree-quantities, such as evolution kernels and matching coefficients.

The hadron matrix elements of the TMD operators with open vector and spinor indices $(1.1,1.2)$ are to be decomposed over all possible Lorentz variants, which define TMD parton distribution functions (TMDPDFs). It is convenient to consider TMD distributions in the impact parameter space, where factorization theorems are naturaly defined. For our purposes we need only a part of the complete decomposition,

$$
\begin{aligned}
& \Phi_{q \leftarrow h, i j}(x, \boldsymbol{b})=\left\langle h\left|\Phi_{i j}(x, \boldsymbol{b})\right| h\right\rangle=\frac{1}{2}\left(f_{1} \gamma_{i j}^{-}+g_{1 L} S_{L}\left(\gamma_{5} \gamma^{-}\right)_{i j}\right. \\
& \left.+\left(S_{T}^{\mu} i \gamma_{5} \sigma^{+\mu}\right)_{i j} h_{1}+\left(i \gamma_{5} \sigma^{+\mu}\right)_{i j}\left(\frac{g_{T}^{\mu v}}{2}+\frac{b^{\mu} b^{v}}{\boldsymbol{b}^{2}}\right) \frac{S_{T}^{v}}{2} h_{1 T}^{\perp}+\ldots\right), \\
& \Phi_{g \leftarrow h, \mu v}(x, \boldsymbol{b})=\left\langle h\left|\Phi_{\mu v}(x, \boldsymbol{b})\right| h\right\rangle \\
& =\frac{1}{2}\left(-g_{T}^{\mu v} f_{1}^{g}-i \varepsilon_{T}^{\mu v} S_{L} g_{1 L}^{g}+2 h_{1}^{\perp g}\left(\frac{g_{T}^{\mu v}}{2}+\frac{b^{\mu} b^{v}}{\boldsymbol{b}^{2}}\right)+\ldots\right),
\end{aligned}
$$

where the vector $b^{\mu}$ is a 4-dimensional vector of the impact parameter space $\left(b^{+}=b^{-}=0\right.$ and $\left.-b^{2}=\boldsymbol{b}^{2}>0\right)$ and $S_{T, L}$ are transverse and longitudinal of the hadron spin vector. In Eqs. $(1.7,1.8)$ we write only the TMD distributions that match the twist-2 integrated distributions. The reported distributions are adressed as helicity $\left(g_{1 L}\right.$ and $\left.g_{1 L}^{g}\right)$, transversity $\left(h_{1}\right)$, pretzelosity $\left(h_{1 T}^{\perp}\right)$ and linearly polarized gluon $\left(h_{1}^{\perp g}\right)$ distributions.

\section{Small- $b$ operator product expansion}

The small- $b$ operator product expansion (OPE) is the relation between TMD operators and lightcone operators. Its leading order can be written as

$$
\begin{aligned}
& \Phi_{i j}(x, \boldsymbol{b})=\left[\left(C_{q \leftarrow q}(\boldsymbol{b})\right)_{i j}^{a b} \otimes \phi_{a b}\right](x)+\left[\left(C_{q \leftarrow g}(\boldsymbol{b})\right)_{i j}^{\alpha \beta} \otimes \phi_{\alpha \beta}\right](x)+\ldots, \\
& \Phi_{\mu v}(x, \boldsymbol{b})=\left[\left(C_{g \leftarrow q}(\boldsymbol{b})\right)_{\mu v}^{a b} \otimes \phi_{a b}\right](x)+\left[\left(C_{g \leftarrow g}(\boldsymbol{b})\right)_{\mu \nu}^{\alpha \beta} \otimes \phi_{\alpha \beta}\right](x)+\ldots,
\end{aligned}
$$

where the symbol $\otimes$ denotes the Mellin convolution in the variable $x$. The matching coefficients $C(\boldsymbol{b})$ are dimensionless, i.e. they depend on $\boldsymbol{b}$ only logarithmically. The power supressed terms, which are studied for the unpolarized case in [15], are represented in the dots. At this order of OPE, the functions $\phi(x)$ are the formal limit of the TMD operators $\Phi(x, \mathbf{0})$. These functions have a decomposition over Lorentz variants analogous to the decomposition shown in Eqs. (1.7, 1.8). The interesting integrated distributions are adressed as helicity $\left(\Delta f_{q, g}\right)$ and transversity $\left(\delta f_{q, g}\right)$ distributions for quarks and gluons.

To study the different matchings one by one we have to project over the corresponding Lorentz variants. We introduce the universal notation

$$
\Phi_{q}^{[\Gamma]}=\frac{\operatorname{Tr}(\Gamma \Phi)}{2}, \quad \Phi_{g}^{[\Gamma]}=\Gamma^{\mu v} \Phi_{\mu v}
$$


Both sides of Eqs. $(2.1,2.2)$ should be supplemented by the ultraviolet renormalization constants. Additionally, the TMD operator is to be multiplied by the rapidity renormalization factor $R$. The renormalized TMD operator is written as

$$
\Phi^{\mathrm{ren}}(x, \boldsymbol{b} ; \mu, \zeta)=Z(\mu, \zeta \mid \varepsilon) R(\boldsymbol{b}, \mu, \zeta \mid \varepsilon, \delta) \Phi(x, \boldsymbol{b} \mid \varepsilon, \delta) .
$$

The renormalization factors do not depend on the Lorentz structure but depend on the parton flavor. The explicit expressions for these factors up to NNLO can be found in $[10,16]$.

The cancellation of rapidity divergences for the spin-dependent distributions is not trivial. If we consider the small- $b$ OPE for a generic quark operator up to NLO,

$$
\begin{aligned}
& \Phi_{q}^{[\Gamma]}=\Gamma^{a b} \phi_{a b}+a_{s} C_{F} \boldsymbol{B}^{\varepsilon} \Gamma(-\varepsilon)[ \\
& -\left(\gamma^{+} \gamma^{-} \Gamma+\Gamma \gamma^{-} \gamma^{+}\right)^{a b}+\bar{x}\left(\frac{g_{T}^{\alpha \beta}}{2}-\frac{b^{\alpha} b^{\beta}}{4 \boldsymbol{B}} \varepsilon\right)\left(\gamma^{\mu} \gamma_{\alpha} \Gamma \gamma_{\beta} \gamma_{\mu}\right)^{a b} \\
& +\left(\frac{1}{(1-x)_{+}}-\ln \left(\frac{\delta}{p^{+}}\right)\right)\left(\gamma^{+} \gamma^{-} \Gamma+\Gamma \gamma^{-} \gamma^{+}+\frac{i \varepsilon \gamma^{+} \not b \Gamma}{2 \boldsymbol{B}}+\frac{i \varepsilon \Gamma \not b \gamma^{+}}{2 \boldsymbol{B}}\right)^{a b} \\
& \left.-\frac{i \pi}{2}\left(\gamma^{+} \gamma^{-} \Gamma-\Gamma \gamma^{-} \gamma^{+}+\frac{i \varepsilon \gamma^{+} \not b \Gamma}{2 \boldsymbol{B}}-\frac{i \varepsilon \Gamma \not b \gamma^{+}}{2 \boldsymbol{B}}\right)^{a b}\right] \otimes \phi_{a b}+\mathscr{O}\left(a_{s}^{2}\right) .
\end{aligned}
$$

where $\boldsymbol{B}=\boldsymbol{b}^{2} / 4>0, a_{s}=g^{2} /(4 \pi)^{d / 2}$, we see that it is affected by logarithms of $\delta$ that represent rapidity divergences. These rapidity divergences are to be eliminated by the $R$ factor which up to NLO reads

$$
R=1+2 a_{s} C_{F} \boldsymbol{B}^{\varepsilon} \Gamma(-\varepsilon)\left(\mathbf{L}_{\sqrt{\zeta}}+2 \ln \left(\frac{\delta}{p^{+}}\right)-\psi(-\varepsilon)-\gamma_{E}\right)+\mathscr{O}\left(a_{s}^{2}\right)
$$

where $\mathbf{L}_{X}=\ln \left(\boldsymbol{B} X^{2} e^{2 \gamma_{E}}\right)$. The rapidity divergence cancels in the product $R \Phi$ if and only if

$$
\gamma^{+} \Gamma=\Gamma \gamma^{+}=0
$$

and if we follow the same procedure for gluons, rapidity divergences only cancels if and only if

$$
\Gamma^{+\mu}=\Gamma^{-\mu}=\Gamma^{\mu+}=\Gamma^{\mu-}=0 .
$$

The conditions in Eqs. $(2.7,2.8)$ are satisfied only for the following Lorentz structures

$$
\Gamma^{q}=\left\{\gamma^{+}, \gamma^{+} \gamma^{5}, \sigma^{+\mu}\right\}, \quad \Gamma^{g}=\left\{g_{T}^{\mu v}, \varepsilon_{T}^{\mu v}, b^{\mu} b^{v} / \boldsymbol{b}^{2}\right\},
$$

which correspond to the Lorentz structures for the so called "leading dynamical twist" TMD distributions. The relations in Eqs. $(2.7,2.8)$ provide a definition of the leading dynamical twist for TMD operators without referring to a particular cross-section. 


\section{Helicity distribution}

In the case of helicity distribution the Lorentz structures for quark and gluon operators are

$$
\Gamma=\gamma^{+} \gamma^{5}, \quad \Gamma^{\mu v}=i \varepsilon_{T}^{\mu \nu}
$$

that depend on the definition of $\gamma_{5}$ matrix in dimensional regularization. The most popular schemes for QCD calculations are 't Hooft-Veltman-Breitenlohner-Maison (HVBM) [17, 18], and Larin scheme $[19,20]$. In both schemes the combination $\gamma^{+} \gamma_{5}$ can be presented as

$$
\gamma^{+} \gamma^{5}=\frac{i}{3 !} \varepsilon^{+v \alpha \beta} \gamma_{v} \gamma_{\alpha} \gamma_{\beta}
$$

where in HVBM scheme the $\varepsilon^{\mu v \alpha \beta}$ is defined only for 4-dimensional set of indices, but in Larin scheme it is different from zero for all set of $d$-dimensional indices. Larin scheme is more convenient than HVBM because it does not violate Lorentz invariance, but for TMD calculations it is inapplicable. The point is that it does violate the leading dynamical twist Eq. (2.7). To ensure that the Eq. (2.7) is fulfilled, we perform a light modification of the Larin scheme, and we call it Larin $^{+}$scheme. In this new scheme, the expression for $\gamma^{+} \gamma_{5}$ is

$$
\left(\gamma^{+} \gamma^{5}\right)_{\text {Larin }^{+}}=\frac{i \varepsilon^{+-\alpha \beta}}{2 !} \gamma^{+} \gamma_{\alpha} \gamma_{\beta}=\frac{i \varepsilon_{T}^{\alpha \beta}}{2 !} \gamma^{+} \gamma_{\alpha} \gamma_{\beta}
$$

The NLO calculation is parallel to unpolarized calculation done in details in [10]. The matching onto integrated distributions is written as

$$
\begin{aligned}
R \Phi_{q}^{\left[\gamma^{+} \gamma_{5}\right]} & =\Delta C_{q \leftarrow q} \otimes \phi_{q}^{\left[\gamma^{+} \gamma_{5}\right]}+\Delta C_{q \leftarrow g} \otimes \phi_{g}^{\left[\varepsilon_{T}\right]} \\
R \Phi_{g}^{\left[\varepsilon_{T}\right]} & =\Delta C_{g \leftarrow q} \otimes \phi_{q}^{\left[\gamma^{+} \gamma_{5}\right]}+\Delta C_{g \leftarrow g} \otimes \phi_{g}^{\left[\varepsilon_{T}\right]}
\end{aligned}
$$

where

$$
\begin{aligned}
& \Delta C_{q \leftarrow q}=\delta(\bar{x})+a_{S} C_{F}\left\{2 \boldsymbol{B}^{\varepsilon} \Gamma(-\varepsilon)\left[\frac{2}{(1-x)_{+}}-2+\bar{x}(1+\varepsilon) \mathscr{H}_{\text {sch. }}+\delta(\bar{x})\left(\mathbf{L}_{\sqrt{\zeta}}-\psi(-\varepsilon)-\gamma_{E}\right)\right]\right\}_{\varepsilon \text {-finite }}, \\
& \Delta C_{q \leftarrow g}=a_{S} C_{F}\left\{2 \boldsymbol{B}^{\varepsilon} \Gamma(-\varepsilon)\left[x-\bar{x} \mathscr{H}_{\text {sch. }}\right]\right\}_{\varepsilon \text {-finite }}, \quad \Delta C_{g \leftarrow q}=a_{S} C_{F}\left\{2 \boldsymbol{B}^{\varepsilon} \Gamma(-\varepsilon)\left[1+\bar{x} \mathscr{H}_{\text {sch. }}\right]\right\}_{\varepsilon \text {-finite }}, \\
& \Delta C_{g \leftarrow g}=\delta(\bar{x})+a_{s} C_{A}\left\{2 \boldsymbol{B}^{\varepsilon} \Gamma(-\varepsilon) \frac{1}{x}\left[\frac{2}{(1-x)_{+}}-2-2 x^{2}+2 x \bar{x} \mathscr{H}_{\text {sch. }}+\delta(\bar{x})\left(\mathbf{L}_{\sqrt{\zeta}}-\psi(-\varepsilon)-\gamma_{E}\right)\right]\right\}_{\varepsilon \text {-finite }},
\end{aligned}
$$

where the subscript " $\varepsilon$-finite" implies the removal of $\varepsilon$-singular terms. The only difference between the results calculated in both schemes is accumulated in the coefficient

$$
\mathscr{H}_{\text {sch. }}=\left\{\begin{array}{cc}
1+2 \varepsilon & \text { HVBM } \\
\frac{1+\varepsilon}{1-\varepsilon} & \text { Larin }^{+} .
\end{array}\right.
$$

One can see that up to $\varepsilon$-supressed parts results in both schemes coindice. The drawback of both schemes of Adler-Bardeem theorem for the non-renormalization of the axial anomaly. This is 
fixed by an extra finite renormalization constant $Z_{q q}^{5}$ from an external condition (see [19, 21, 22]). In the TMD framework, the condition is the requirement of equality between helicity and unpolarized matching coefficients.

$$
\left[Z_{q q}^{5}(\boldsymbol{b}) \otimes \Delta C_{q \leftarrow q}(\boldsymbol{b})\right](x)=C_{q \leftarrow q}(x, \boldsymbol{b}) .
$$

We find the following result for $Z_{q q}^{5}$ up to NLO,

$$
Z_{q q}^{5}=\delta(\bar{x})+2 a_{s} C_{F} B^{\varepsilon} \Gamma(-\varepsilon)\left(1-\varepsilon-(1+\varepsilon) \mathscr{H}_{\text {sch. }}\right) \bar{x} .
$$

For further discussion about $Z_{q q}^{5}$ for TMDs and for the expressions of the matching coefficients in the limit $\varepsilon \rightarrow 0$ see [13].

\section{Transversity and pretzelosity distributions}

At leading twist there is not mixture between quarks and gluons, and the Lorentz structure fot the transversity TMD operator is commonly chosen as $\Gamma=\sigma^{+\mu}$, instead of the more general $\Gamma=i \gamma_{5} \sigma^{+\mu}$, which depend on the scheme. In this case, we find that both the transversity and the pretzelosity distributions have the leading twist- 2 matching on the integrated transversity PDF. Their matching coefficients, $\delta C_{q \leftarrow q}$ and $\delta^{\perp} C_{q \leftarrow q}$ respectively, are defined as

$$
R \Phi_{q}^{\left[\sigma^{+\mu}\right]}=g_{T}^{\mu v} \delta C_{q \leftarrow q} \otimes \phi_{q}^{\left[\sigma^{+v}\right]}+\left(\frac{b^{\mu} b^{v}}{b^{2}}+\frac{g_{T}^{\mu \nu}}{2(1-\varepsilon)}\right) \delta^{\perp} C_{q \leftarrow q} \otimes \phi_{q}^{\left[\sigma^{+v}\right]} .
$$

For the transversity distribution we have up to NLO

$$
\delta C_{q \leftarrow q}=\delta(\bar{x})+a_{s} C_{F}\left\{2 \boldsymbol{B}^{\varepsilon} \Gamma(-\varepsilon)\left[\frac{2}{(1-x)_{+}}-2+\bar{x} \frac{\varepsilon^{2}}{1-\varepsilon}+\delta(\bar{x})\left(\mathbf{L}_{\sqrt{\zeta}}-\psi(-\varepsilon)-\gamma_{E}\right)\right]\right\}_{\varepsilon \text {-finite }}
$$

and for the pretzelosity distribution

$$
\delta^{\perp} C_{q \leftarrow q}=-4 a_{s} C_{F} \boldsymbol{B}^{\varepsilon} \Gamma(-\varepsilon) \bar{x} \varepsilon^{2} .
$$

We can see that the pretzelosity matching coefficient is proportional to $\varepsilon$, i.e. zero. This result coincides with the estimation made in [23]. This observation is indeed supported by the measurements of $\sin \left(3 \phi_{h}-3 \phi_{s}\right)$ asymmetries by HERMES and COMPASS (see [24] and references within). Calculations at NNLO suggest that at this order the pretzelosity is different from zero and it means that would be a quantity that begins at NNLO in QCD.

\section{Conclusions}

In this paper we show the complete set of NLO TMD matching coefficients of the TMD distributions to the twist- 2 integrated distributions in the regime of small- $b$. As we have shown the expressions of the matching coefficients with its complete $\varepsilon$-dependence, the path to the calculations of spin dependent TMDs at NNLO is open and in progress [25]. 
As a consecuence of the evaluation of the OPE for a general operator the possible Lorentz structures for TMDs are restricted, giving the so called "leading dynamical twist" TMD distributions. We also provide a discussion on the different schemes used to deal with the calculation with $\gamma_{5}$ and $\varepsilon_{T}$ in dimensional regularization. Within this discussion the Larin ${ }^{+}$scheme is defined, as a modification of the classical Larin scheme that fulfills the leading dynamical twist conditions. Up to NLO there is not difference between schemes in the calculation of the matching coefficients.

Finally, we obtain that the pretzelosity distribution has $\varepsilon$-supressed matching coefficient, but it is not zero at NNLO. It is a natural explanation of its smallness in phenomenological analyses.

\section{Acknowledgements}

D.G.R. and I.S. are supported by the Spanish MECD grant FPA2014-53375-C2-2-P and FPA2016-75654-C2-2-P. D.G.R. and I.S. are also sponsored by the group UPARCOS.

\section{References}

[1] M. G. Echevarria, A. Idilbi and I. Scimemi, JHEP 1207 (2012) 002 doi:10.1007/JHEP07(2012)002 [arXiv:1111.4996 [hep-ph]].

[2] J. Collins, (Cambridge monographs on particle physics, nuclear physics and cosmology. 32)

[3] J. Y. Chiu, A. Jain, D. Neill and I. Z. Rothstein, JHEP 1205 (2012) 084 doi:10.1007/JHEP05(2012)084 [arXiv:1202.0814 [hep-ph]].

[4] M. G. Echevarria, A. Idilbi and I. Scimemi, Phys. Rev. D 90 (2014) no.1, 014003 doi:10.1103/PhysRevD.90.014003 [arXiv:1402.0869 [hep-ph]].

[5] T. Becher and M. Neubert, Eur. Phys. J. C 71 (2011) 1665 doi:10.1140/epjc/s10052-011-1665-7 [arXiv:1007.4005 [hep-ph]].

[6] S. M. Aybat and T. C. Rogers, Phys. Rev. D 83 (2011) 114042 doi:10.1103/PhysRevD.83.114042 [arXiv:1101.5057 [hep-ph]].

[7] I. O. Cherednikov and N. G. Stefanis, Nucl. Phys. B 802 (2008) 146 doi:10.1016/j.nuclphysb.2008.05.011 [arXiv:0802.2821 [hep-ph]].

[8] I. O. Cherednikov and N. G. Stefanis, Phys. Rev. D 77 (2008) 094001 doi:10.1103/PhysRevD.77.094001 [arXiv:0710.1955 [hep-ph]].

[9] M. G. Echevarria, I. Scimemi and A. Vladimirov, Phys. Rev. D 93 (2016) no.1, 011502 Erratum: [Phys. Rev. D 94 (2016) no.9, 099904] doi:10.1103/PhysRevD.93.011502, 10.1103/PhysRevD.94.099904 [arXiv:1509.06392 [hep-ph]].

[10] M. G. Echevarria, I. Scimemi and A. Vladimirov, JHEP 1609 (2016) 004 doi:10.1007/JHEP09(2016)004 [arXiv:1604.07869 [hep-ph]].

[11] T. Gehrmann, T. Luebbert and L. L. Yang, JHEP 1406 (2014) 155 doi:10.1007/JHEP06(2014)155 [arXiv:1403.6451 [hep-ph]].

[12] T. Gehrmann, T. Lubbert and L. L. Yang, Phys. Rev. Lett. 109 (2012) 242003 doi:10.1103/PhysRevLett.109.242003 [arXiv:1209.0682 [hep-ph]]. 
[13] D. Gutiérrez-Reyes, I. Scimemi and A. A. Vladimirov, Phys. Lett. B 769 (2017) 84 doi:10.1016/j.physletb.2017.03.031 [arXiv:1702.06558 [hep-ph]].

[14] M. G. A. Buffing, M. Diehl and T. Kasemets, arXiv:1708.03528 [hep-ph].

[15] I. Scimemi and A. Vladimirov, JHEP 1703 (2017) 002 doi:10.1007/JHEP03(2017)002 [arXiv:1609.06047 [hep-ph]].

[16] M. G. Echevarria, I. Scimemi and A. Vladimirov, Phys. Rev. D 93 (2016) no.5, 054004 doi:10.1103/PhysRevD.93.054004 [arXiv:1511.05590 [hep-ph]].

[17] G. 't Hooft and M. J. G. Veltman, Nucl. Phys. B 44 (1972) 189. doi:10.1016/0550-3213(72)90279-9

[18] P. Breitenlohner and D. Maison, Commun. Math. Phys. 52 (1977) 11. doi:10.1007/BF01609069

[19] S. A. Larin, Phys. Lett. B 303 (1993) 113 doi:10.1016/0370-2693(93)90053-K [hep-ph/9302240].

[20] S. A. Larin and J. A. M. Vermaseren, Phys. Lett. B 259 (1991) 345. doi:10.1016/0370-2693(91)90839-I

[21] Y. Matiounine, J. Smith and W. L. van Neerven, Phys. Rev. D 58 (1998) 076002 doi:10.1103/PhysRevD.58.076002 [hep-ph/9803439].

[22] V. Ravindran, J. Smith and W. L. van Neerven, Nucl. Phys. B 682 (2004) 421 doi:10.1016/j.nuclphysb.2004.01.001 [hep-ph/0311304].

[23] A. Bacchetta, D. Boer, M. Diehl and P. J. Mulders, JHEP 0808 (2008) 023 doi:10.1088/1126-6708/2008/08/023 [arXiv:0803.0227 [hep-ph]].

[24] C. Lefky and A. Prokudin, Phys. Rev. D 91 (2015) no.3, 034010 doi:10.1103/PhysRevD.91.034010 [arXiv:1411.0580 [hep-ph]].

[25] D. Gutiérrez-Reyes, I. Scimemi and A. A. Vladimirov, Work in progress 